\title{
Endosteal Fibular Strut Graft with Proximal Humeral Locking Plate in Delayed and Neglected Fractures of the Proximal Humerus
}

\author{
Piyush Wasudeo Gadegone, Wasudeo Gadegone*, Vijayanand Lokhande \\ Department of Orthopaedics \& Traumatology, Chandrapur Multispeciality Hospital, Chandrapur, India \\ Email: *gadegone123@yahoo.co.in
}

How to cite this paper: Gadegone, P.W., Gadegone, W. and Lokhande, V. (2020) Endosteal Fibular Strut Graft with Proximal Humeral Locking Plate in Delayed and Neglected Fractures of the Proximal Humerus. Open Journal of Orthopedics, 10 , 359-370.

https://doi.org/10.4236/ojo.2020.1012034

Received: September 18, 2020

Accepted: December 7, 2020

Published: December 10, 2020

Copyright $\odot 2020$ by author(s) and Scientific Research Publishing Inc. This work is licensed under the Creative Commons Attribution International License (CC BY 4.0).

http://creativecommons.org/licenses/by/4.0/

(c) (i) Open Access

\begin{abstract}
Purpose: The purpose of this study was to define a treatment protocol in which a non-vascularized endosteal fibular strut graft, a corticocancellous grafts and a locking plate construct are used for stabilization of the delayed and neglected proximal humerus fractures and to report its outcome. Patients \& Methods: Eleven patients (6 females and 5 males) with delayed, neglected proximal humerus fractures were included in this study, conducted between March 2015 and December 2019. Average age of the patients was 57 years (range: 41 to $67 \mathrm{yrs}$ ). All patients were treated with the debridement, decortication and shingling of the bone at the site of the fracture followed by using an endosteal fibular strut graft, corticocancellous bone grafts and stabilization with locking plate. The patients were followed up for a mean time of 16.3 months (range: 13 to 40 months). The patient outcomes were evaluated using the Disabilities of the Arm, Shoulder, and Hand Questionnaire, and the modified scoring system of Constant and Murley. Results: Union at the fracture site was achieved in all patients at a mean of 8.5 months (range: 6 to 11 months). The DASH score improved from an average pre-operative score of 71.1 (range: 64 to 78 ) to an average post-operative score of 25.2 (range: 21 to 35 ) at the final follow-up. Albeit with a small sample size of $n=11$, this difference was found to be statistically significant $(\mathrm{p}<0.05)$. The CM score improved from an average pre-operative score of 33.2 (range: 20 to 48 ) to an average post-operative score of 66.8 (range: 59 to 72 ) at the final follow-up. This difference was also found to be statistically significant in this patient cohort $(\mathrm{p}<0.05)$. The results were excellent in 3 patients, good in 6 and moderate in 2. Conclusions: An endosteal fibular strut, subperiosteally placed cortico-cancellous grafts with a locking plate fixation helps in biological healing of neglected fractures of proximal humerus.
\end{abstract}




\section{Keywords}

Proximal Humerus, Endosteal Fibular Graft, Neglected Fractures, Locking

Plate, Cortico-Cancellous Bone Grafts

\section{Introduction}

The incidence of proximal humeral fractures accounts for $5 \%$ to $8 \%$ of all fractures [1]. In developing countries, ignorance and lack of accurate diagnosis lead to fractures being neglected for a long period of time. The proximal humerus fractures, after initial neglect, are extremely challenging to treat because they are often associated with shoulder and elbow joint stiffness, marked osteopenia and metaphyseal or diaphyseal bone defects [2]. To date, there is no consensus on a standardized treatment or management protocol of neglected fractures of the humerus. Many surgeons agree that in neglected and nonunited fracture cases in young adults, salvage is possible and an attempt should be made to preserve the humeral head and native shoulder joint be means of novel fixation techniques [3]. Various methods of bone grafting are described in the literature. The fibula is a long bone that can be useful as vascularized or non-vascularized graft in reconstruction of bony defects [4]. Non-vascularized fibula should be considered a valuable alternative treatment option for patients with bone defects or segmental reconstructions. The use of fibular strut bone graft, the stability of fixation, stiffness of the plate and screw constructs are markedly improved [5]. Patient outcomes i.e. safety and effectiveness of the procedure in allowing a patient to regain the ability to function and perform activities of daily living in a near pain-free state similar to their pre-fracture state, depend highly on the integrity and quality of the fracture union [6]. The locking plate with autogenous grafting is accepted as the gold standard method which has yielded satisfactory results in nonunited fractures.

The goals of this article were: 1) to define an open reduction treatment protocol in which a non-vascularized endosteal fibular strut graft, a supplementary cortico-cancellous iliac crest graft and a locking plate construct are used for stabilization and osteosynthesis of the neglected proximal humerus fracture, and 2) to report on patient outcomes, i.e. safety and effectiveness data, following this treatment protocol.

\section{Patients and Methods}

This study was conducted at a multispecialty orthopaedic hospital and government medical college following receipt of approval from the hospital's human research ethics committee. A written informed consent was obtained from all patients who agreed to participate in this study. A total of 11 patients (six female and five male) were included in this study that was conducted between March 2015 and December 2019 at a multispecialty orthopaedic hospital and govern- 
ment medical college. Inclusion criteria were neglect of at least more than four weeks in seeking care at our institution with fractures of the proximal humerus.

Average patient age at the time of surgery was 57 years (range: 41 to 67 years). Proximal humerus fractures of delayed presentation and neglected are included in the study without extension into the head of the humerus.

Six patients received initial treatment from a village Hakim (i.e. a local practitioner "specializing" in traditional medicine). Five patients were considered as being negligent or ignorant as they received either no care or were taken to local "bone-setters". The average time of neglect from initial injury for all 11 patients in this study was 7 weeks (range: 4 to 12 weeks). All patients presented with closed fractures, and upon further evaluation revealed radiological findings like an atrophic type of non-union. The patients reported mild pain, displayed tenderness and abnormal mobility at the site of fracture, and complained of difficulty in performing basic activities of daily living.

\subsection{Pre-Operative Evaluation \& Fracture Classification}

Pre-operative radiographic evaluation was conducted in all patients. For all patients, anterior posterior (AP) radiographic views were taken with both internal and external rotation of the shoulder joint, however a radiograph in the axillary view position was not possible in some patients because of shoulder stiffness. All patients were observed to have stiffness of their shoulder and elbow joints to varying degrees. All the necessary investigations were completed to rule out the possibility of an infection.

With regards to treatment options for fractures of the proximal humerus, various classification schemes have been proposed, however, there is no classification scheme described for neglected fracture of humerus. Using the existing classification scheme presented by Checcia and colleagues [7] based on the criteria of nonunion and the characteristic of fracture pattern, five patients in our study were classified to be in Group 1, four patients in Group 2 and two patients in Group 3.

\subsection{Surgical Technique}

All the patients agreed to the written consent after explaining them about the procedure.

A delto-pectoral approach was used to expose the fracture site. Excision of the fibrous and devitalized bone was done. The medullary canal was opened with straight and curved bone awl and if needed canal was opened with appropriate reamer to accommodate the fibular strut graft. Shingling of proximal and distal part of fracture site was done for cortico-cancellous grafts. Under Tourniquet control the midshaft of the fibula was harvested with care taken to protect the superficial peroneal nerve. We resorted to harvest a slightly lengthy fibula so that it could be trimmed as necessary to telescope snugly into the fragments across the fracture site and proximally into hollow cavity of proximal humerus [Figure 1(a), Figure 1(b)]. 


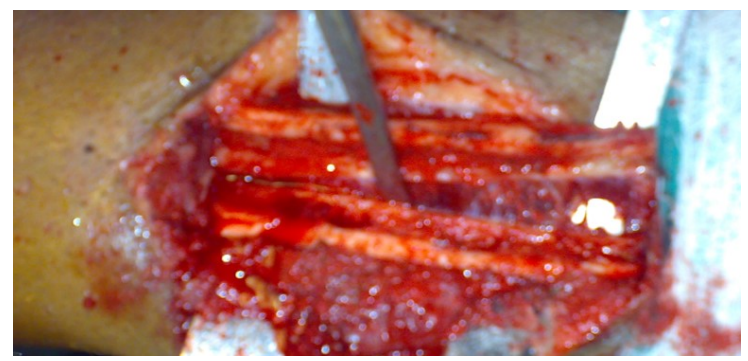

(a)

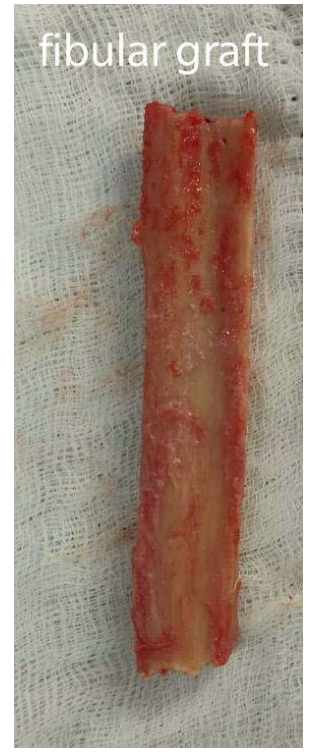

(b)

Figure 1. (a) Harvesting of fibular graft; (b) Fibular strut graft.

Once the final shaping of the graft and length was ascertained, the fracture was reduced. The intramedullary fibular graft was placed in the medullary cavity across the fracture site and proximally it extended into the humeral head. A Philos plate was used for the fixation of the fracture. The care was taken to incorporate fibula in screw fixation to increase the strength of the plate screw construct. The corticocancellous grafts were filled in the surrounding area of the fracture site. The wound was closed in layers over a negative suction drain. All the patients received three doses of intravenous Inj. Cephalosporin 1.5 gms and Inj Amikacin 500 mgs according to the hospital policy.

The Shoulder and elbow exercises were initiated a day after the operation depending upon the patient comfort. The sutures were removed after 10 days. Three patients had superficial infection. They were controlled in five days with dressing and antibiotics. The patients were guided by the physiotherapy protocols to be followed. After hospital discharge, the patients were observed on a monthly basis until healing of the fracture clinically and radiologically. The presence of bridging callus, as well as any loosening or failure of the fixation was noted. The fracture union was considered, if on X-ray, the callus formation was seen in three of four cortices on AP and lateral views. 


\subsection{Clinical Scores and Statistics}

Data was collected in this study retrospectively. Two well established and frequently used clinical scoring methods were used i.e. DASH Questionnaire (Disabilities of the Arm, Shoulder and Hand) and the CMS (Constant \& Murley Score) [8]. DASH provides data on grip strength, range of motion, radiographic parameters and functional outcomes whereas CMS objectively evaluates shoulder outcomes. Statistical analyses were conducted on the data collected to test the null hypothesis at an $\alpha$ of 0.05 , the null hypothesis being that there would be no statistically significant difference between the preoperative and post-operative DASH and CMS (Table 1).

\section{Results}

Complete union of the fracture was successfully achieved in all patients at a mean follow-up time of 8.5 months (range: 6 to 11 months). This was confirmed upon clinical evaluation as well as radiographic determination of bridging calluses observed between the fractured bone segments.

Table 1. Data analysis DASH and CMS score.

\begin{tabular}{|c|c|c|c|c|}
\hline Patient No. & $\begin{array}{c}\text { Preoperative } \\
\text { DASH }\end{array}$ & $\begin{array}{c}\text { Postoperative } \\
\text { DASH }\end{array}$ & $\begin{array}{c}\text { Preoperative } \\
\text { CMS }\end{array}$ & $\begin{array}{c}\text { Postoperative } \\
\text { CMS }\end{array}$ \\
\hline 1 & 77 & 25 & 37 & 72 \\
\hline 2 & 68 & 21 & 25 & 64 \\
\hline 3 & 78 & 23 & 28 & 72 \\
\hline 4 & 68 & 25 & 36 & 72 \\
\hline 5 & 68 & 21 & 48 & 62 \\
\hline 6 & 69 & 24 & 20 & 59 \\
\hline 7 & 76 & 25 & 28 & 60 \\
\hline 8 & 74 & 22 & 35 & 68 \\
\hline 9 & 72 & 35 & 44 & 70 \\
\hline 10 & 6 & 42 & 43 & 668 \\
\hline 11 & 6 & 83 & 22 & 868 \\
\hline Average & 71.1 & 25.2 & 33.2 & 66.8 \\
\hline Std. Dev. & 4.6 & 4.4 & 8.3 & 4.8 \\
\hline Minimum & 64 & 21 & 20 & 59 \\
\hline Maximum & 78 & 35 & 48 & 72 \\
\hline
\end{tabular}


The average DASH score changed from a pre-operative value of 71.1 (SD: 4.6; range: 64 to 78 ) to a post-operative value of 25.2 (SD: 4.4; range: 21 to 35 ) at the final follow-up. A paired t-test for two sample groups was conducted to determine statistical significance at $\alpha=0.05$. The p-value was observed to be significantly less than 0.05 , i.e. $\mathrm{p}<0.05$. The null hypothesis was therefore rejected, indicating that there was detection of a statistically significant difference between the means of the pre-operative and post-operative data groups. Post-operatively, the average DASH score reduced by $64.5 \%$, which also met the criteria of a clinically significant difference. On similar lines, the average CMS changed from a pre-operative value of 33.2 (SD: 8.3; range: 20 to 48 ) to a post-operative value of 66.8 (SD: 4.8; range: 59 to 72) at the final follow-up. Again, a paired t-test for two sample groups was conducted to determine statistical significance at $\alpha=$ 0.05 . The $\mathrm{p}$-value was observed to be significantly less than 0.05 , i.e. $\mathrm{p}<0.05$. There was detection of a statistically significant difference between the means of the preoperative and post-operative data groups. Post-operatively, the average CMS more than doubled, i.e. an increase of $>100 \%$, which too met the criteria of a clinically significant difference (Table 2 and Table 3 ).

Table 2. t-Test: paired two samples for means COMPARISON OF DASH.

\begin{tabular}{ccc}
\hline & Preoperative DASH & Postoperative DASH \\
\hline Mean & 71.09090909 & 25.18181818 \\
Variance & 20.89090909 & 19.56363636 \\
Observations & 11 & \\
Pearson Correlation & -0.005845844 & \\
Hypothesized Mean Difference & 0 & \\
df & 10 & \\
\hline
\end{tabular}

$\mathrm{P}<0.05$. This difference is considered to be statistically significant therefore the null hypothesis is rejected.

Table 3. t-Test: paired two samples for means COMPARISON OF CMS.

\begin{tabular}{ccc}
\hline & Preoperative CMS & Postoperative CMS \\
\hline Mean & 33.18181818 & 66.81818182 \\
Variance & 69.16363636 & 23.36363636 \\
Observations & 11 & \\
Pearson Correlation & 0.32678795 & \\
Hypothesized Mean Difference & 0 & \\
Df & 10 & \\
\hline
\end{tabular}

$\mathrm{P}<0.0001$. This difference is considered to be statistically significant therefore the null hypothesis is rejected. 
This bar graph shows a comparison between the average preoperative and postoperative DASH scores and CMS (chart).

Objective functional outcomes, i.e. range of motion and basic tasks when conducting activities of daily living, using a patient satisfaction rating were also recorded and reported for all patients. These were observed to be excellent in three patients, good in six patients and moderate in two patients. None of the patients reported as having poor functional outcomes following this procedure.

There were no perioperative complications noted for any of the patients. Postoperatively, one patient developed transient radial nerve palsy, which completely recovered six months post-operatively. Two patients reported discomfort over the fibular graft harvest site with weakness in peroneal compartment, which recovered at the six-month follow-up period. One patient who had posterior fracture dislocation prior to the surgery had developed avascular necrosis following the surgery. However, this patient was able to conduct routine activities of daily living with minimal pain. None of the patients presented with any progressive loosening or mechanical failure of the PHILOS locking plate and screw components (Figures 2(a)-(d)), (Figures 3(a)-(d)), (Figures 4(a)-(d)).

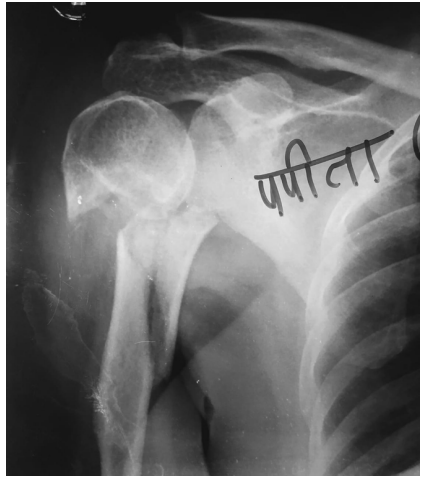

(a)

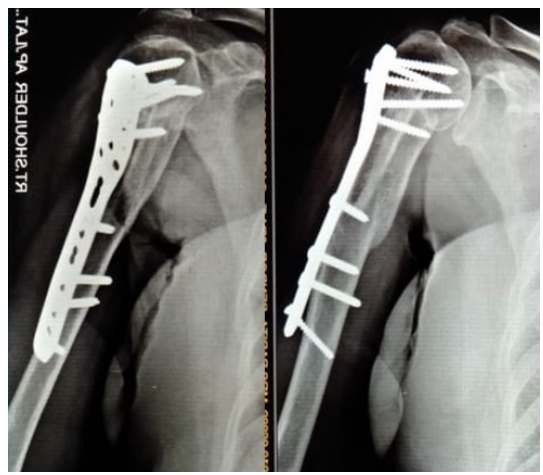

(c)

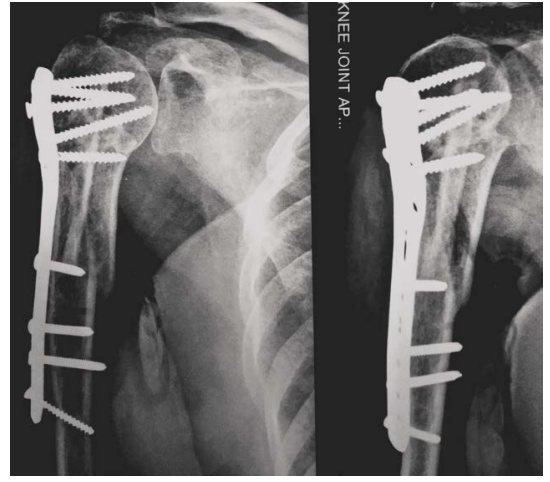

(b)

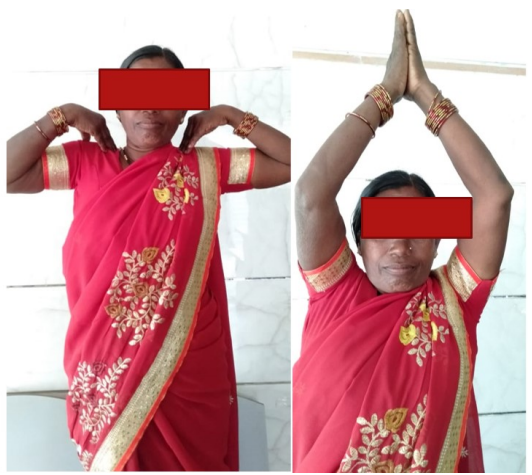

(d)

Figure 2. (a) Neglected fracture proximal humerus with medial comminution with osteopania; Two months old neglected fracture proximal humerus in a $44 \mathrm{yr}$ female labour by occupation; (b) X-ray after three months osteosynthesis with strut nonvascularised fibular graft, cancellous bone grafting surrounding the fracture site and locking compression plate; (c) Three years of follow-up with good radiological union and functional outcome; (d) Follow up clinical picture. 


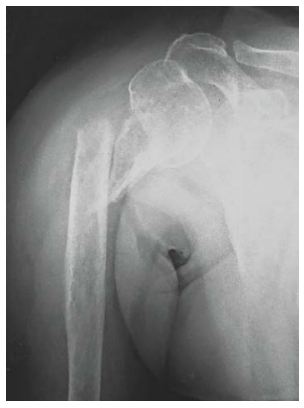

(a)

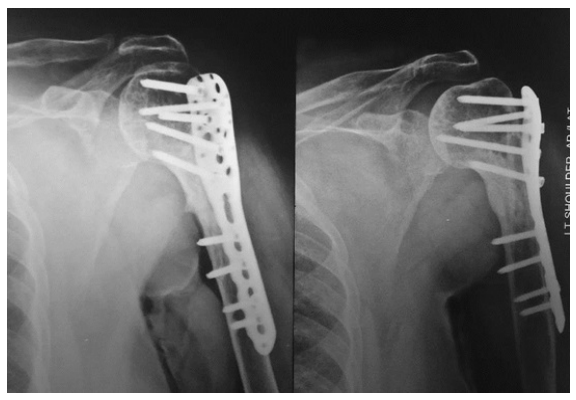

(c)

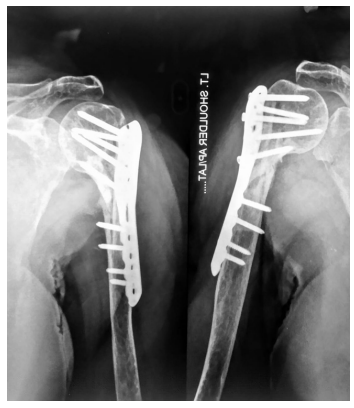

(b)

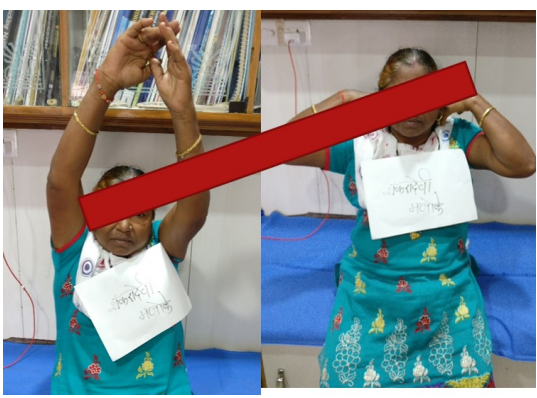

(d)

Figure 3. (a) 68 YR female with diabetes 12 weeks neglected proximal humerus fracture with deficient lateral wall; (b) X-ray after three months. osteosynthesis with strut non vascularised fibular graft, cancellous bone grafting surrounding the fracture site and locking compression plate; (c) Three years of follow up with good radiological union and functional outcome; (d) Follow up clinical picture.

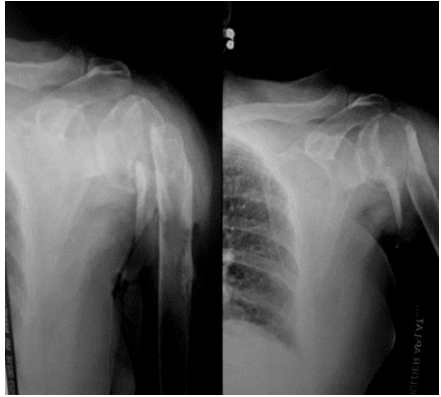

(a)

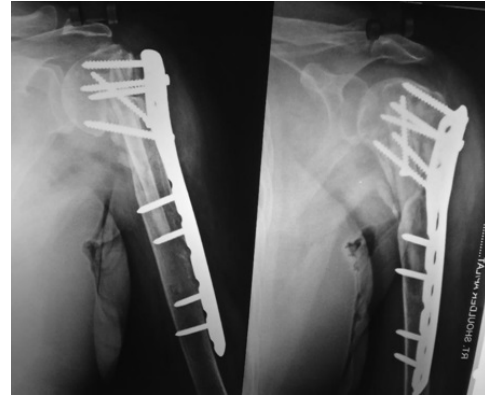

(b)

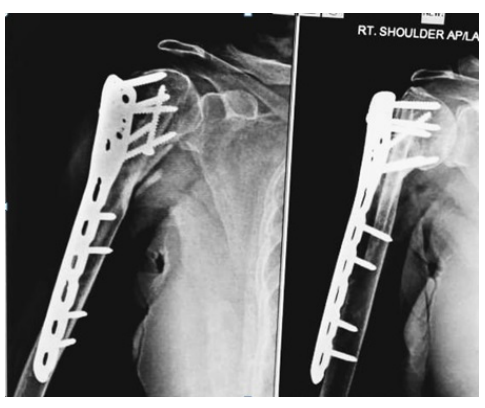

(c)

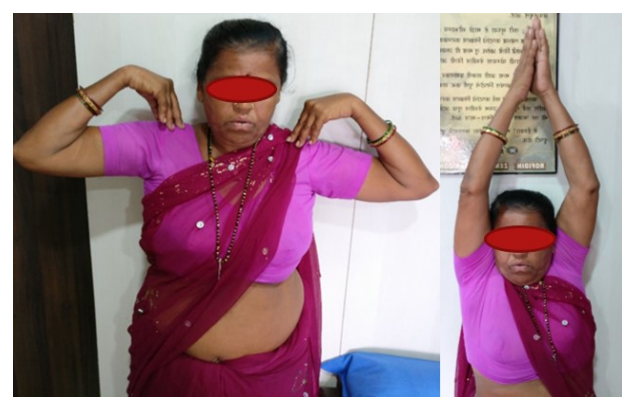

(d)

Figure 4. (a) Fourteen weeks old neglected proximal humerus fracture in $52 \mathrm{yr}$ old female. Doubtful viability, osteoporosis; (b) X-ray after two months. Osteosynthesis with strut nonvascularised fibular graft and locking compression plate extensive cancellous bone grafting surrounding the fracture site; (c) One year of follow up with good radiological union and functional outcome; (d) Follow up clinical picture. 


\section{Discussion}

The term neglected, in the context of seeking medical treatment, is generally defined as the passage of a time period of at least three weeks since the occurrence of an injury for which there is either no treatment sought or a lack of appropriate and adequate treatment administered. Neglected proximal humerus fractures pose a challenging problem to the treating orthopaedic surgeon as there is a dearth of published literature that provides a preferred treatment protocol or procedure option that has been successful in patients such cases [9]. Delayed and neglected fractures often lead to added complications including stiffness of the shoulder and elbow joints, osteopenia or bone loss or both. In addition, the ends of the fractured bones may become atrophic, which could hinder the process of osteosynthesis and make fracture fixation challenging. Time based changes in the physical and biological properties of bones in the cases of neglected fractures make it difficult to select any particular treatment of choice since it is difficult to gauge whether any type of implant hardware will be able to sustain the loads at the fracture site without being subject to failure, especially during the healing process of first four to six weeks during which the patients are advised to start rehabilitation [10].

A multitude of treatment methods are described in the literature for the treatment of failed fracture fixation cases (i.e. cases of nonunion or malunion) of the proximal humerus including intramedullary nailing, plate fixation with or without bone grafting and prosthetic replacement [11]. However, there are no standard protocols or recommended treatments for delayed and neglected fractures of the proximal humerus. Owing to their similarities to cases of delayed union, nonunion, some of those techniques can be successfully employed for the treatment of neglected fractures. The goal of this article was to propose a treatment protocol and evaluate its outcome on a small group of patients that met the inclusion criteria of neglected fracture cases of the proximal humerus.

Successful surgical management of any humeral nonunion or malunion case requires a stable internal fixation that enables early joint motion. An autologous bone graft is also advocated to promote the natural bone healing process in such cases [12] [13]. Due to the continual advent of newer operative techniques and implant designs in the management of complex fracture cases, a stable internal fixation is now easily achievable even in the most complex of cases.

In this research study we followed the general principles of achieving a stable internal fixation in which we used an autologous midshaft non-vascularized fibular strut graft as an internal splint in combination with autologous iliac cortico-cancellous bone chips and a PHILOS bone plate and screw construct to treat complicated and neglected cases of proximal humeral fractures. In this treatment protocol, the cortical fibular graft provides increased mechanical strength to the fixation in which it withstands significant multi-axial loads, while simultaneously transferring higher forces to the surrounding native bone than would be possible with using only a bone plate construct. This enables quicker healing 
of the fracture in accordance with Wolfe's law. The addition of autologous corticocancellous bone at the fracture site aids the fracture healing process by simulating the biological cascade of bone healing. In its entirety, the fixation construct recommended by us in this treatment protocol ensures that the bones at the fracture site, both the native and autografts, are adequately stressed, and have sufficient strength to handle the observed forces to allow for a strong, stable and successful healing of the fracture. In this study, following the aforementioned treatment protocol, all patients with neglected fractures of the proximal humerus progressed to complete union without any implant or bone related failure. One patient developed avascular necrosis of the humeral head and refused a shoulder arthroplasty since the patient was able to carry out activities of daily living with mild pain and limited shoulder movements.

The results of this study are difficult to compare with the results currently published in literature because most of the studies report on cases of nonunion or malunion following failure of the primary fracture fixation methods or implant hardware related failure. Nonetheless, our results are still comparable to such published data to some extent. In one such study, a union rate of $96 \%$ was achieved with plate fixation in combination with intramedullary bone peg insertion and cancellous bone grafting [13]. The rate of complications reported in that study was also low. A union rate of $100 \%$ was reported in another article on nonunion fracture cases [12]. The neglected fractures mimics like nonunited fractures hence it requires the procedure which will produce early union of atrophic nonunited bone and to restore the strength of functionless limb. Our procedure helps in salvaging the fracture by achieving early union. It has profound effect on the overall compliance of the patient, reduces the repeated morbidity from the lengthy and costly treatment of prosthetic replacement. We did not see any complication like the postoperative infection; implant cut through and even fracture of the fibula graft in any case. Transient radial nerve palsy was noted in one patient which might be during the tissue handling at the time of fixation, and it recovered fully after six months of follow up. Two patients had donor site discomfort and peroneal compartment weakness resolved after physiotherapy in three months.

Hertel et al. [14] in their follow up study observed that initial predictors of humeral head ischemia do not necessarily preclude development of avascular necrosis and therefore, fixation is worth considering in such cases even if signs of ischemia are present [15].

Successful surgical management of delayed and neglected proximal humeral fractures require stable internal fixation with osteoinductive and osteoconductive bone grafts for early union that would enable pain free and greater range of early joint motion. The use of our treatment protocol led to a $100 \%$ rate of union in the patients included this study. The fixation construct used herein, i.e. a PHILOS plate with a nonvascularized endosteal fibular graft with corticocancellous packing, was sufficiently rigid to prevent progressive loosening or brea- 
kage of the implant hardware. The functional and radiographic results we report are both encouraging and promising. Based on our observation of the patient outcomes with this treatment protocol, we wish to recommend it as a gold standard of treatment for neglected fractures of the proximal humerus. It is also noted that if such a reconstruction is not possible or is tried and fails, performing a traditional or reverse shoulder arthroplasty still remains available as the next in line viable option for these patients [16].

This study isn't without its limitations. Our patient cohort was very small in size comprising of only 11 patients. The follow-up duration was also less than 24 months, which is short-term. As a result, we are unaware of the long-term implications of this treatment protocol and if it will continue to allow the patients to live a close to normal and pain free lifestyle as it relates to their shoulder joint mobility. Lastly, we could not locate any other peer reviewed published articles that report on patient outcomes in specific cases of neglected fractures of the proximal humerus, therefore, we could not adequately compare the findings of our study to similarly published data. Longer term studies including a greater number of patients are required to corroborate the findings of our study, and we encourage the orthopaedic surgeon community to implement the treatment protocol we recommend and publish their findings in-line with the data we report.

\section{Conclusion}

An endosteal fibular strut graft provides added stability of fixation with a locking plate for treatment of delayed and neglected fractures of the proximal humerus. Additionally, subperiosteally placed corticocancellous grafts at the fracture fixation site provide for a quick response time the biological healing of the bone. The treatment procedure defined in this study can be universally adopted for a successful outcome in cases of delayed and neglected fractures of the long bones.

\section{Conflict of Interest}

The authors certify that there are no conflicts of interest with any organization regarding the subject matter of this article. No direct or indirect benefit was received or will be received in connection with the publication of this research study.

\section{References}

[1] Volgas, D.A., Stannard, J.P. and Alonso, J.E. (2004) Nonunions of the Humerus. Clinical Orthopaedics and Related Research, 419, 46-50. https://doi.org/10.1097/00003086-200402000-00008

[2] Court-Brown, C.M. and McQueen, M.M. (2008) Nonunions of the Proximal Humerus: Their Prevalence and Functional Outcome. Journal of Trauma and Acute Care Surgery, 64, 1517-1521. https://doi.org/10.1097/TA.0b013e3181469840

[3] Cheung, E.V. and Sperling, J.W. (2008) Management of Proximal Humeral Nonunions and Malunions. Orthopedic Clinics of North America, 39, 475-482. 
https://doi.org/10.1016/j.ocl.2008.06.002

[4] Jupiter, J.B. (1990) Complex Non-Union of the Humeral Diaphysis: Treatment with a Medial Approach, an Anterior Plate, and a Vascularized Fibular Graft. The Journal of Bone and Joint Surgery, 72, 701-707. https://doi.org/10.2106/00004623-199072050-00009

[5] El-Sayed, M., El-Hadidi, M. and El-Adl, W. (2007) Free Non-Vascularised Fibular Graft for Treatment of Post-Traumatic Bone Defects. Acta Orthopaedica Belgica, 73, 70-76.

[6] Pugh, D.M. and McKee, M.D. (2003) Advances in the Management of Humeral Nonunion. Journal of American Academy of Orthopaedic Surgeons, 11, 48-59. https://doi.org/10.5435/00124635-200301000-00007

[7] Checcia, S.L., Doneux, P., Miyazaki, A.N., Spir, I.A., Bringel, R. and Ramos, C.H. (2000) Classification of Nonunions of the Proximal Humerus. International Orthopaedics, 24, 217-220. https://doi.org/10.1007/s002640000157

[8] Constant, C.R. and Murley, A.H. (1987) A Clinical Method of Functional Assessment of the Shoulder. Clinical Orthopaedics and Related Research, 10214, 160-164. https://doi.org/10.1097/00003086-198701000-00023

[9] Lu, Y., Jiang, C., Zhu, Y., Wang, M., Bowles, R.J. and Mauffrey, C. (2014) Delayed ORIF of Proximal Humerus Fractures at a Minimum of 3 weeks from Injury: A Functional Outcome Study. European Journal of Orthopaedic Surgery and Traumatology, 24, 715-721. https://doi.org/10.1007/s00590-013-1255-5

[10] Duralde, X.A. and Leddy, L.R. (2010) The Results of ORIF of Displaced Unstable Proximal Humeral Fractures Using a Locking Plate. Journal of Shoulder and Elbow Surgery, 19, 480-488. https://doi.org/10.1016/j.jse.2009.08.008

[11] Galatz, L.M. and Iannotti, J.P. (2000) Management of Surgical Neck Nonunions. Orthopedic Clinics of North America, 31, 51-61. https://doi.org/10.1016/S0030-5898(05)70127-9

[12] Badman, B.L., Mighell, M., Kalandiak, S.P. and Prasarn, M. (2009) Proximal Humeral Nonunions Treated with Fixed-Angle Locked Plating and an Intramedullary Strut Allograft. Journal of Orthopaedic Trauma, 23, 173-179. https://doi.org/10.1097/BOT.0b013e31819b0bdc

[13] Walch, G., Badet, R., Nové-Josserand, L. and Levigne, C. (1996) Nonunions of the Surgical Neck of the Humerus: Surgical Treatment with an Intramedullary Bone Peg, Internal Fixation, and Cancellous Bone Grafting. Journal of Shoulder and Elbow Surgery, 5, 161-168. https://doi.org/10.1016/S1058-2746(05)80001-1

[14] Hamilton, S.W. and Baird, K.S. (2009) The Treatment of Established Non-Union of the Proximal Humerus Using the Polarus Locking Intramedullary Nail. International Journal of Shoulder Surgery, 3, 53-56. https://doi.org/10.4103/0973-6042.59970

[15] Hertel, R., Hempfing, A., Stiehler, M. and Leunig, M. (2004) Predictors of Humeral Head Ischemia after Intracapsular Fracture of the Proximal Humerus. Journal of Shoulder and Elbow Surgery, 13, 427-433. https://doi.org/10.1016/j.jse.2004.01.034

[16] Lenze, U., Pohlig, F., Knebel, C., Lenze, F., Harrasser, N., Mühlhofer, H., Toepfer, A., Rechl, H. and von Eisenhart-Rothe, R. (2017) Autologous Fibula Transplantation for Reconstruction of Bone Defects. Orthopade, 46, 648-655. https://doi.org/10.1007/s00132-017-3442-2 